Faculty of Veterinary Medicine,

AL-Baath University, Syria.

\title{
BLOOD LEAD LEVELS IN COWS REARED AROUND POLLUTED LOCALITIES AND ITS INFLUENCE ON MILK LEAD LEVELS
}

(With 4 Tables)

By

\section{AL-AKOUN; M. AMMAR NABHAN and A. AL-AWAD (Received at 22/11/2011)}

مستويات الرصاص في دم الأبقار المرباة حول المناطق المعرضة للتثلوث

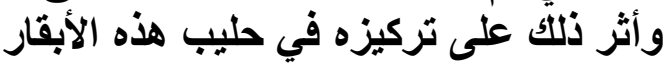

مصطفى العكون ، محد عمار نبهان ، عو/د العو/د

يعتبر الرصاص ملوثاً بيئياً واسع الانتشار له مخاطر صحية كثيرة على صحة الإنسان

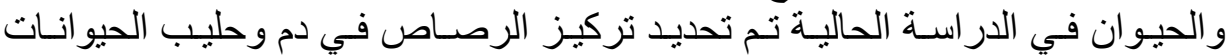

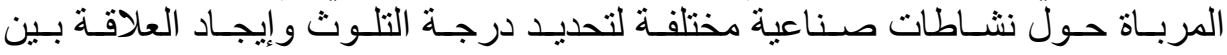

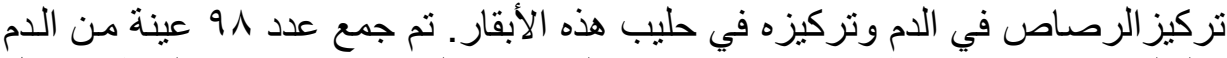

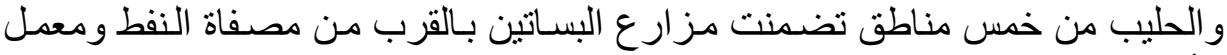

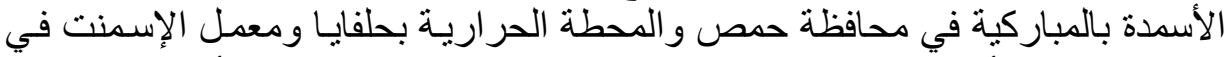

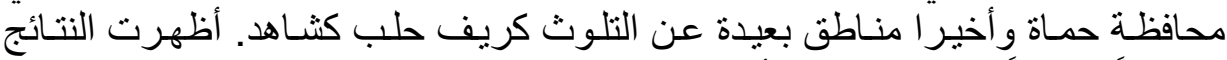

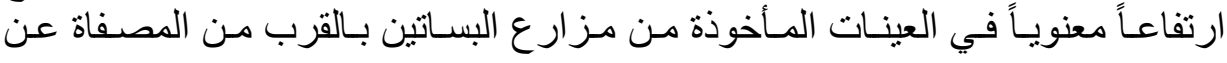

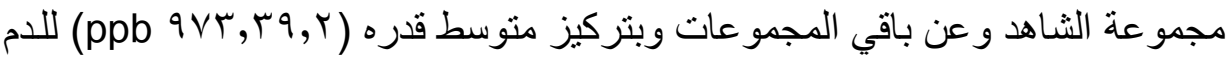

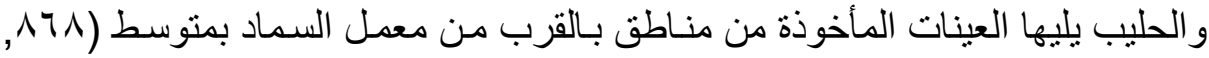

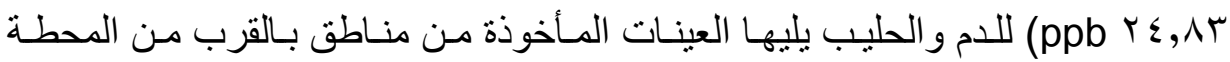
الحرارية بحلفايا وبتركيز متوسط ( • بم , r r ppb) للام و الحليب على الترتيب يليها

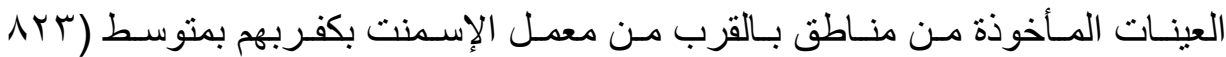

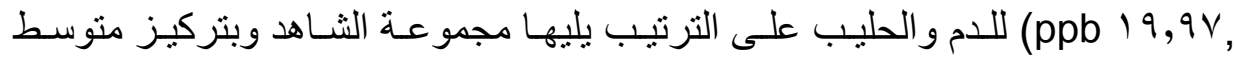
للدم و الحليب على الترتيب. أمسا بالنسـبة للعلاقـة بـين تركيـز (ppb IV , \& , VTV)

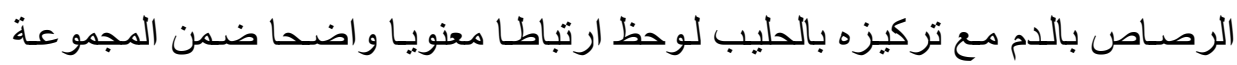




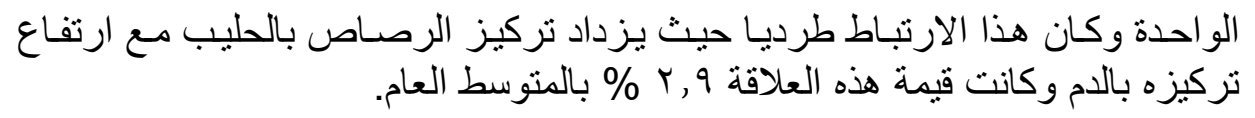

\section{SUMMARY}

Lead is pervasive environmental pollutant with potential public health hazard. The present study examines the blood and milk lead level in animals reared in areas around different industrial activities to determine the degree of pollutant and to find the relationship between blood and milk lead levels. The milk and blood samples $(n=98)$ were collected from animals reared around five area, al-psateen farms near the oil refinery (homs) and al-moubarakiea near chemical fertilizer factory (homs) and heat-electricity station (hulfaia-hama) and cement factory (kafer-bu hama) and finally country of Aleppo serve as controls. Significantly $(\mathrm{P}<0.05)$ higher milk and blood lead levels was recorded in animals reared around al-psateen farms near the oil refinery (the average concentration 39.2 - $973 \mathrm{ppb}$ ) for milk and blood followed by al-moubarakiea near chemical fertilizer factory plant (the average concentration $24.83-868 \mathrm{ppb}$ ) for milk and blood followed by heatelectricity station (hufaia-hama) (the average concentration $23-830 \mathrm{ppb}$ ) for milk and blood followed by cement factory (kafer-bu hama) (the average concentration $19.97-823 \mathrm{ppb}$ ) for milk and blood and and finally control group (the average concentration 17.4 -737 ppb) for milk and blood. Concerning the obtained results showed high positive correlation in the same group (lead concentration increase in blood so increase in milk) the average value of this relation were $2.9 \%$.

Key words: Lead, cows, milk, blood.

\section{INTRODUCTION}

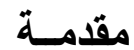

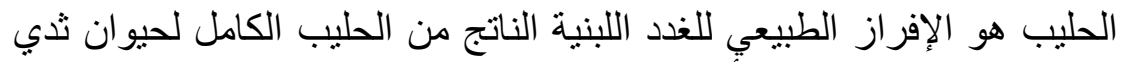

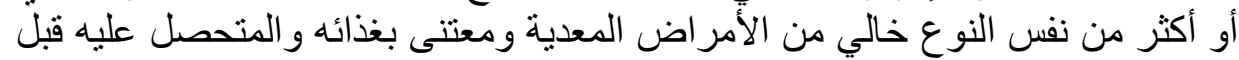

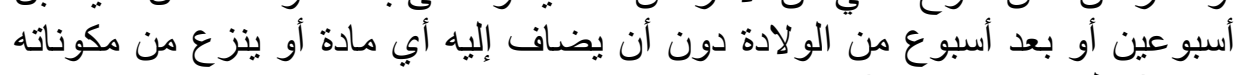

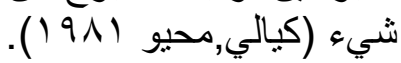




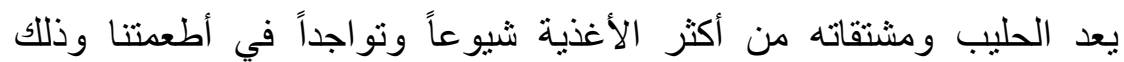

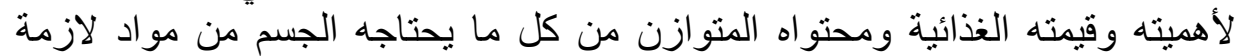

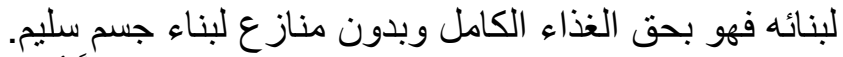

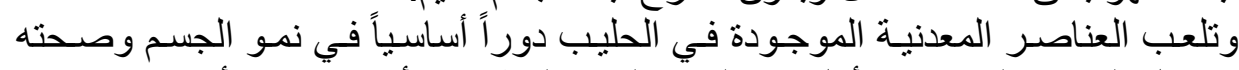

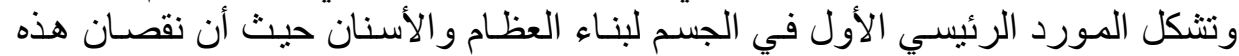

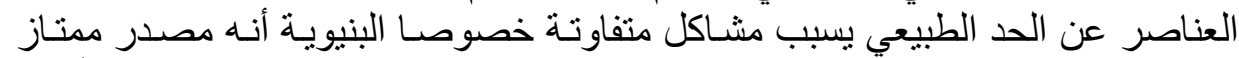

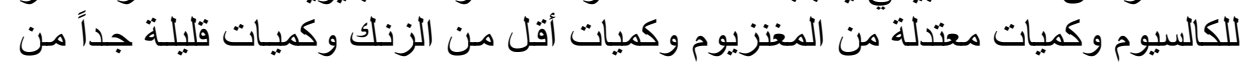
الحديد والنحاس (Levy et al., 1985; Pennigton et al., 1995)

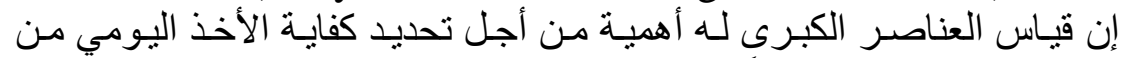

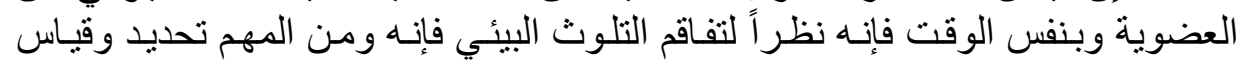

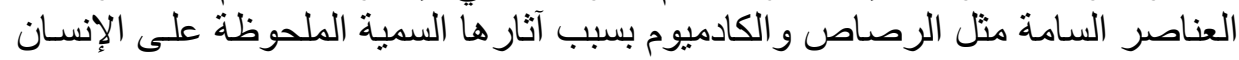

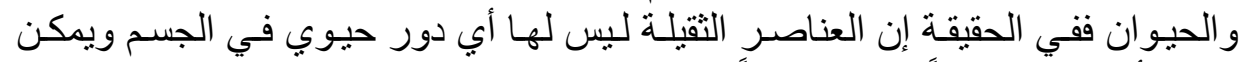

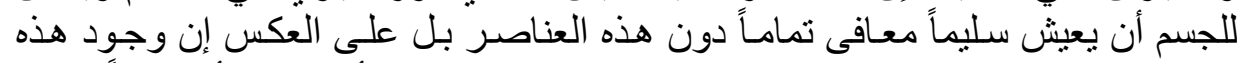

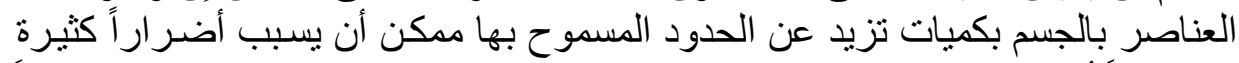

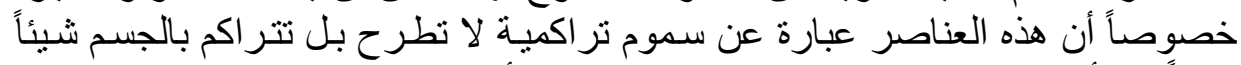

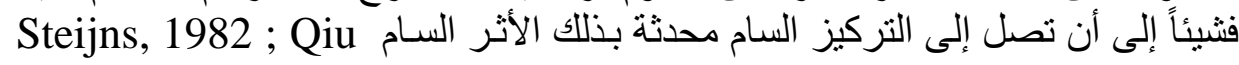
(et al., 2009) ولعلنا لانكون مبالغين إذا قلنا إن أهم وأخطر هذه العناصر هو العنصر الذي يتمحور عليه بحثنا وهو الرصناص.

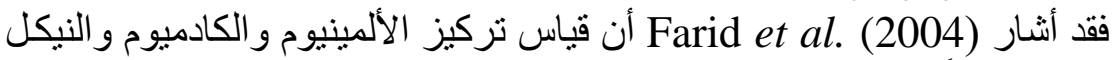
و الرصاص مهم جداً في حليب الأبقار بسبب سميتها.

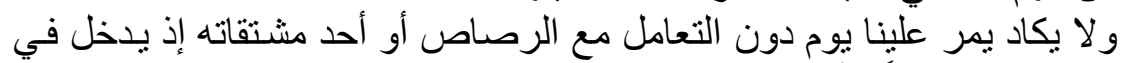

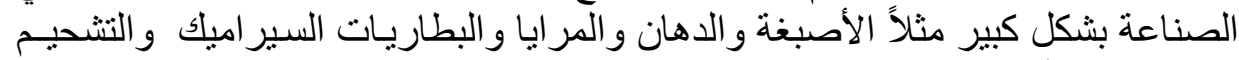

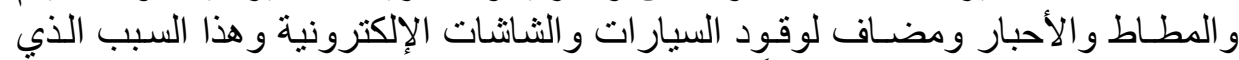

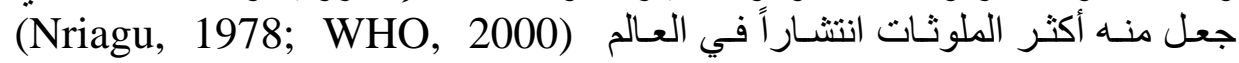

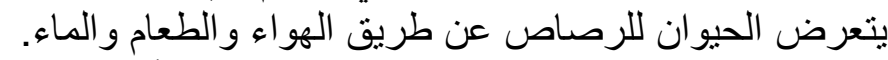

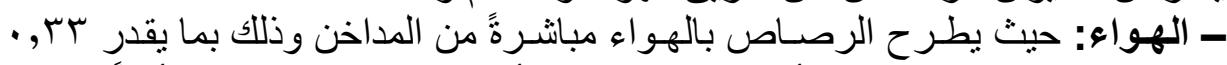

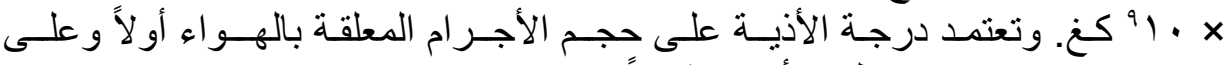

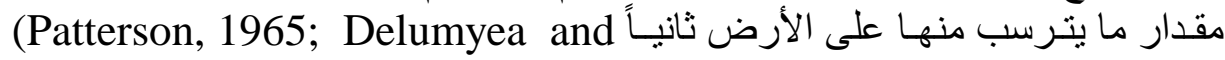
Kalivretenos, 1987; Nriagu and Pacyna, 1988; Ducoffre et al., 1990)

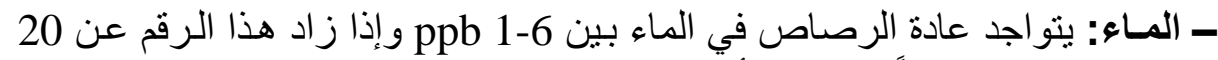

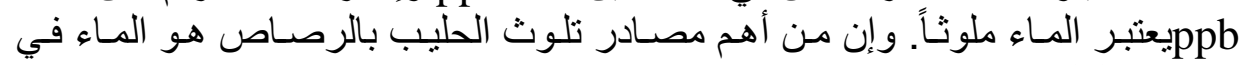
المناطق الملوثة (Kramer et al., 1994).

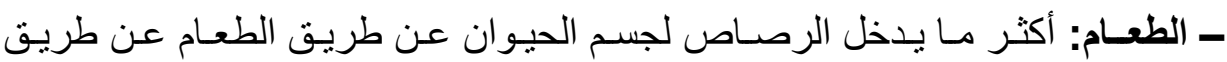

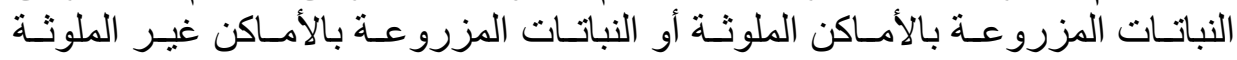


و المعرضة للتلوث مثل طرق النقل السريع إذ يصل تركيز الرصـاص فيها أعلى بعشر مرات عن القيم الطبيعية (العواد ,2008).

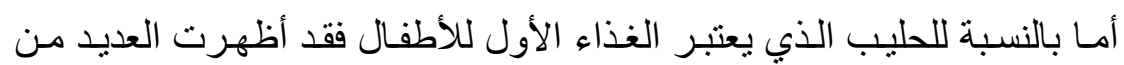

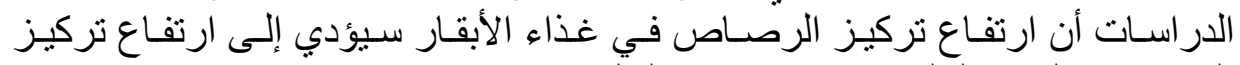

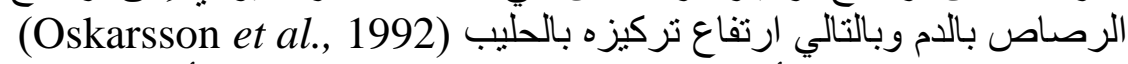

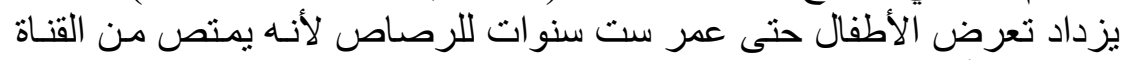

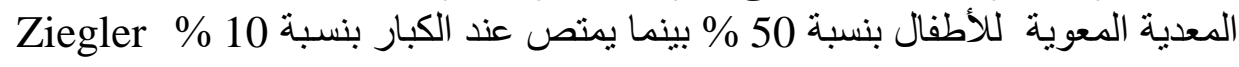
.et al., 1978)

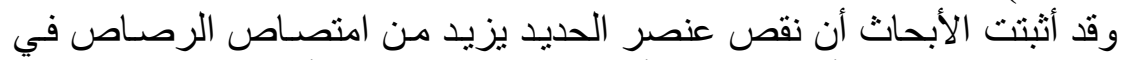

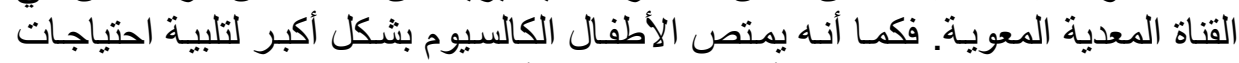

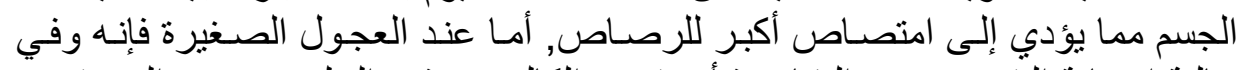

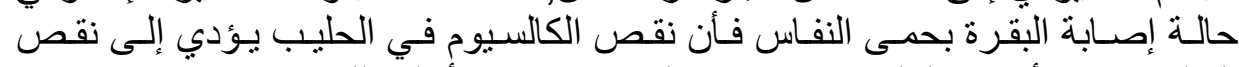

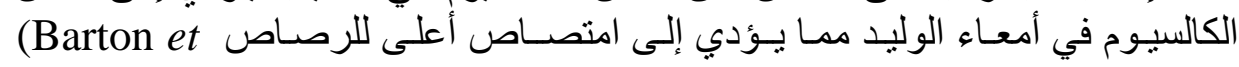
al., 1978) قامت منظمة الغذاء و الدو اء الأمريكية بتحديد الحد اليومي المسموح لدخول الرصاص إلى الجسم حتى 90 ميكرو غرام اللإلبالغين.

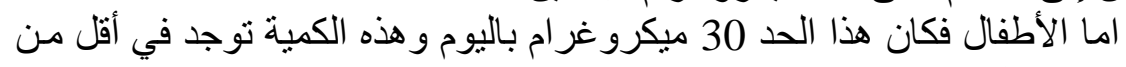
نصف ليتر من الحليب في منطقة قليلة التلوث

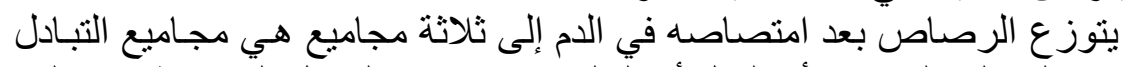

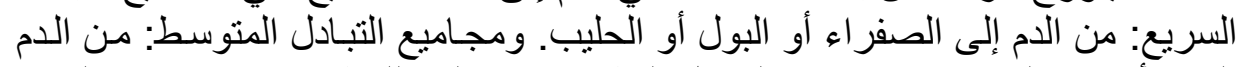

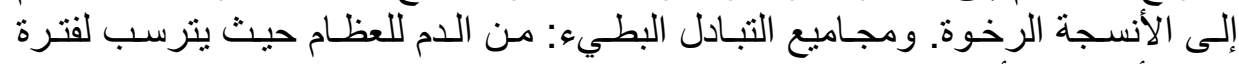

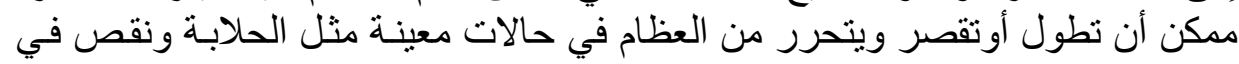

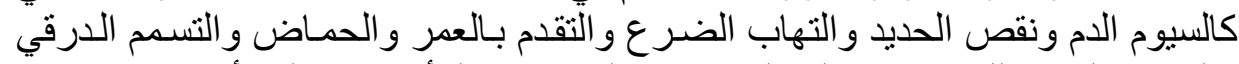

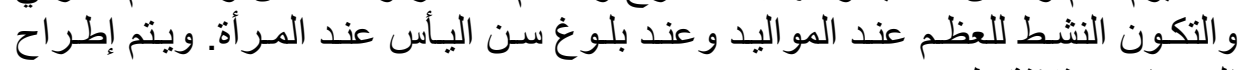

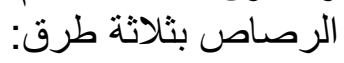

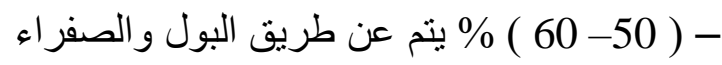

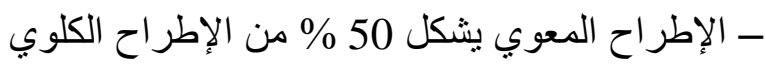

- يفرز عن طريق الحليب 10 \% من الرصاص الإط المنص (WHO, 2001)

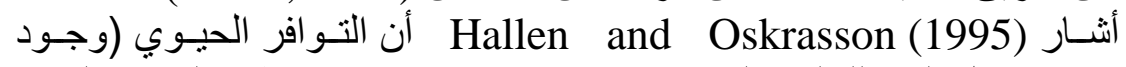

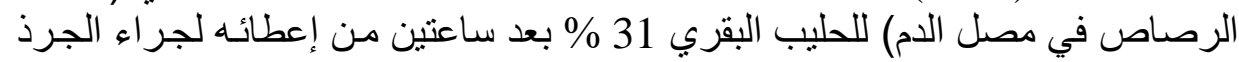

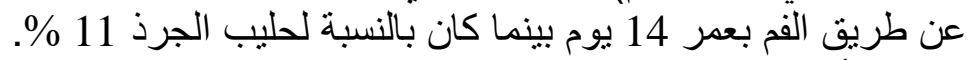

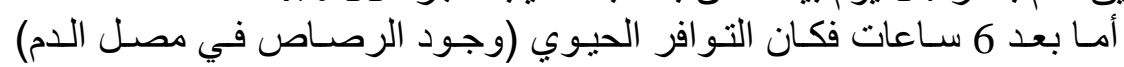

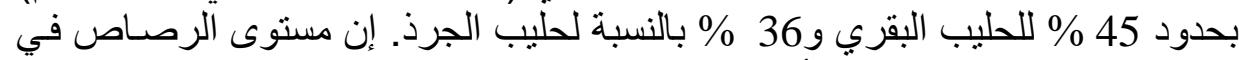

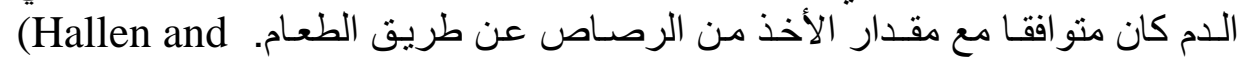
Oskrasson, 1995) 


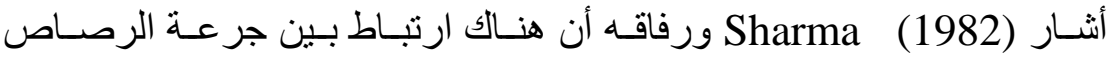

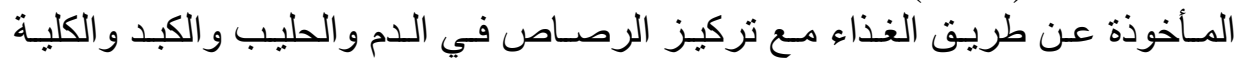

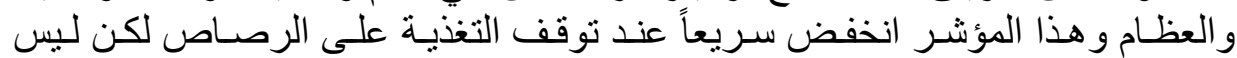
بالعظام (Sharma et al., 1982).

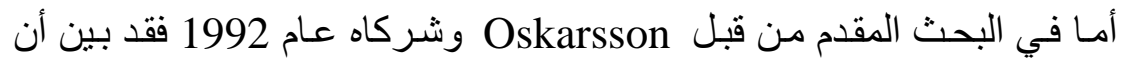

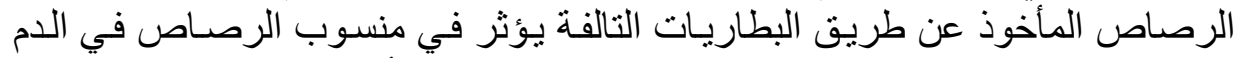

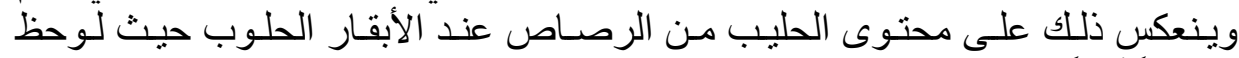
ارتفاعاً أسياً بين منسوب الرصاص في الدم و الحليب (Oskarsson et al., 1992)

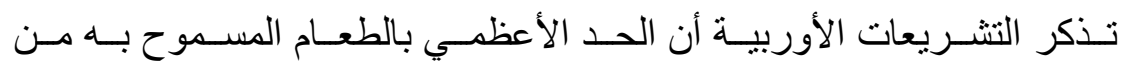

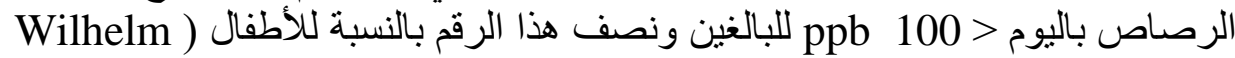
.(et al., 1995

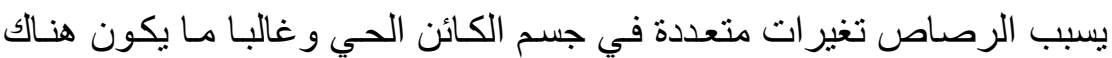

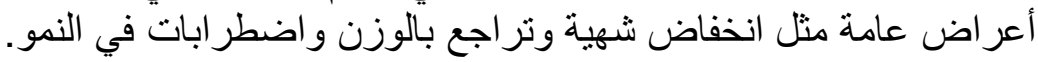
تشير الأبحاث أن العلاقة بين نسبة الرصساص في الحليب إلى نسبة الرصساص أنسا

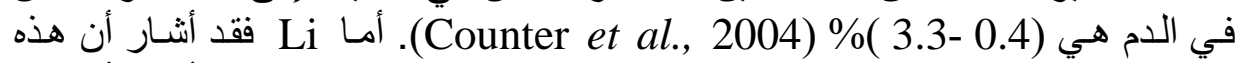

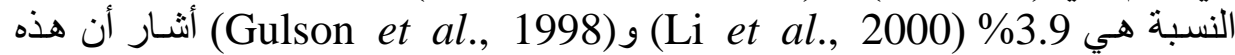

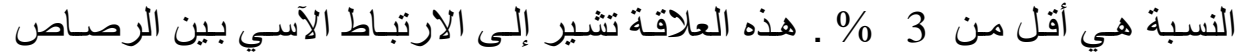

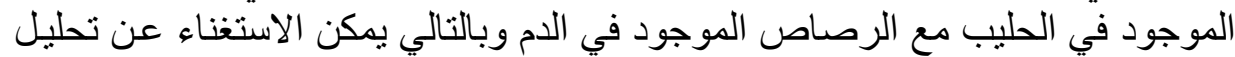
رصاص الدم (Gulson et al., 1998).

أهداف البحث : (أبث: ـ الكثف عن عنصـر الرصـاص في حليب ودم الأبقار حول المنـاطق الصناعية مـن المنطقة الوسطى.

ـ مقارنة النتائج مع عينات حليب ودم من مناطق بعيدة نسبياً عن أماكن التلوث.

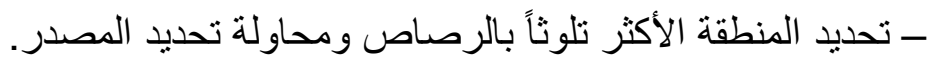
- إيجاد العلاقة التي تحدد نسبة الرصاص في الدم إلى نسبة الرصاص بالحليب.

\section{MATERIALS and METHODS}

مواد وطرائق البحث

أولا : المواد اللازمة لإجراء البحث : 


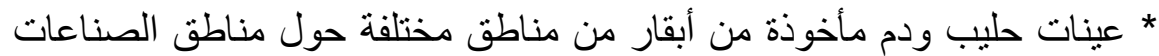

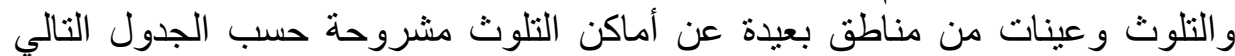

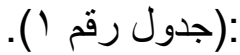

جدول رقم ا : يوضح أماكن جمع العينات ومصادر التلوث المحتملة

\begin{tabular}{|c|c|c|c|c|c|}
\hline المنطقة|لخامسة & المنطقة|لر ابعة & المنطقةالثالثة & المنطقة/الثانية & المنطقة|الولى & \\
\hline ريف حلب & المباركية & البساتين & كفربهم & حلفايا & المكان \\
\hline حلب & حمص & حمص & حماة & حماة & المحافظة \\
\hline لايوجد & معمل الأسمدة & مصفاة النفط & الإسمنت & الحرارية & التلوث \\
\hline r. & 19 & 19 & YI & $r$. & العينات \\
\hline- & أقل من اكم معل & آكم حولة & أقل من بولم معمل & حول المح منة & البعدعن \\
\hline (الثـاهد) & الر ابعة & الثالثة & الثانية & الأولى & المجموعة \\
\hline
\end{tabular}

* جهاز مطياف الامتصاص الذري/20mic absorption spectrometry

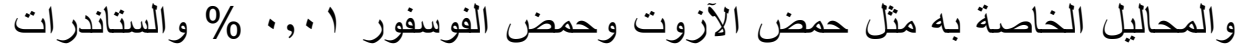
المعيارية الخاصة بالرصاص بله وتريتون 100 - X وأمونيوم ديهايدروجين فوسفات

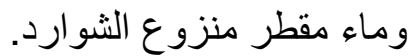
* أنابيب بلاستيكية محكمة الإغلاق لجمع عينات الحليب سعة 10 مل مل وأنابيب

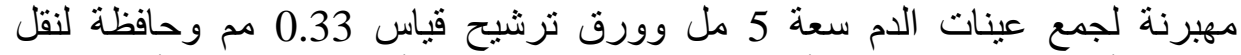
العينات أثناء الجمع ومجمدة أفقية بدرجة ملة - 20 مئوية وأقماع فصل من أجل فصل فئل

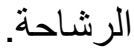
طريقة البحث: تم جمع 99 عينة قسمت إلى خمس مجمو عات تبعا لمناطق جمع العينات وهي: - عينات حليب ودم لنفس الحيوان وبنفس اللحظة أخذت من الأبقار المرباة في ريف

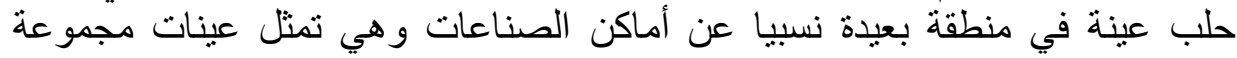
الثناهد - عينات حليب ودم لنفس الحيوان وبنفس اللحظة أخذت من الأبقار المرباة حول المحطة الحرارية بحلفايا عينة في محافظة حماة وهي تمثل المجمو عة الأولى. 
- عينات حليب ودم لنفس الحيوان وبنفس اللحظة أخذت من الأبقار المرباة حول

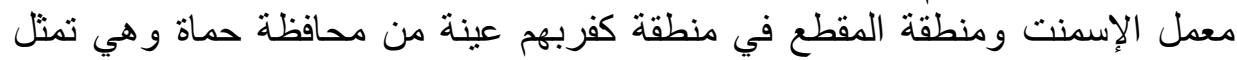
المجموعة الثنانية.

ـ عينات حليب ودم لنفس الحيوان وبنفس اللحظة أخذت من الأبقار المرباة في منطقة

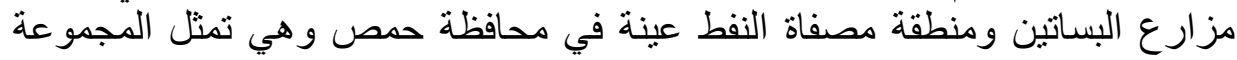

- ع عينات حليب ودم لنفس الحيوان وبنفس اللحظة أخذت من الأبقار المرباة في منطقة

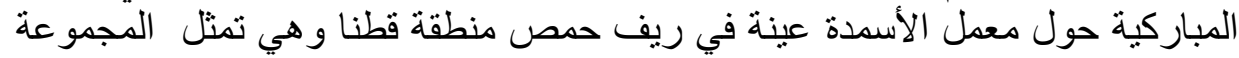

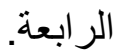

تم جمع العينات في الفترة الواقعة بين الثهر الثالث والرابع من العام 2011 والثة

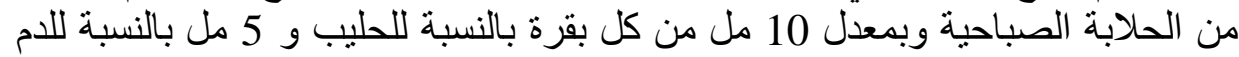

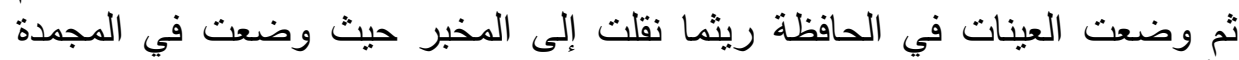

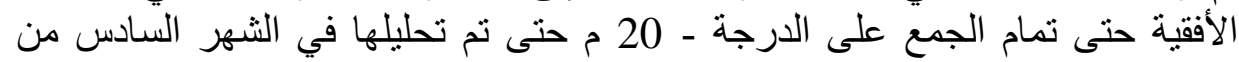
العام 2011 .

تم تحليل العينات لتحديد تركيز الرصاص باستخدام جهاز مطياف الامتصاص

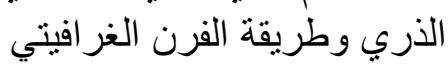

Graphite furnace atomic absorption spectrometry (GFAAS)

باستخدام لمبة وحيدة الطيف حسب التعليمات التي وصفها (Jeng et al., 1994) وفقا

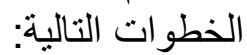

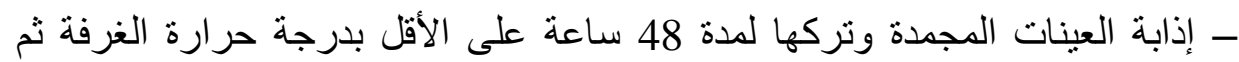
التأكد من انخفاض حموضة العينات حتى PH=4.5 ثم الترشيح وتحة التحليل الرشاحة. ـ تحضير المحلول الخاص لتحليل مصل الحليب والمكون من: 3 أحجام من ماء

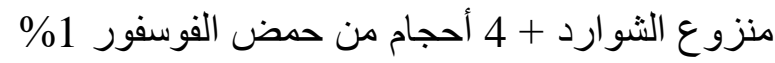

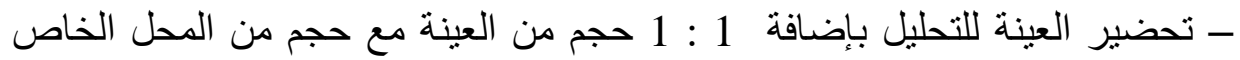

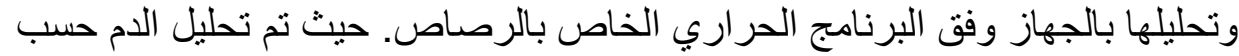

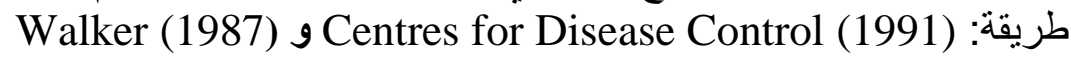

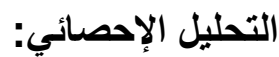
تم باستخدام برنامج Statistical وطريقة One way ANOVA

\section{RESULTS and DISCUSSION النتائج والمناقشة}

أظهرت نتائج التحليل الكيميائي للحليب أن العينات المأخوذة من منطقة مزارع الأرئ

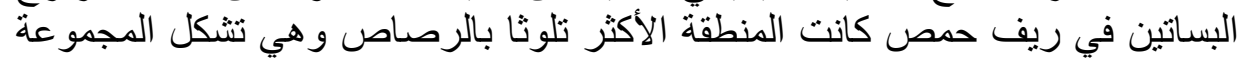

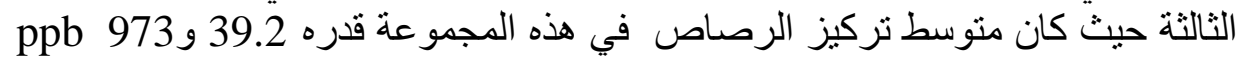




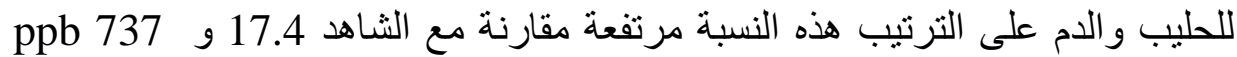

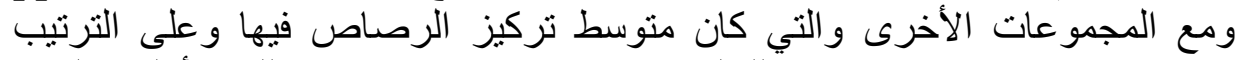

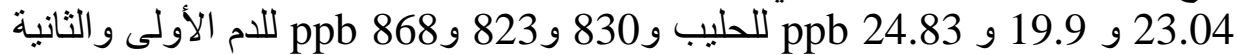

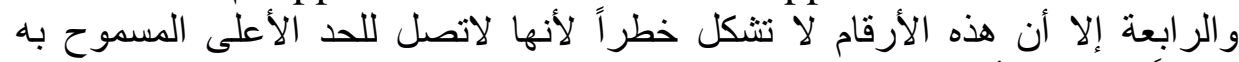

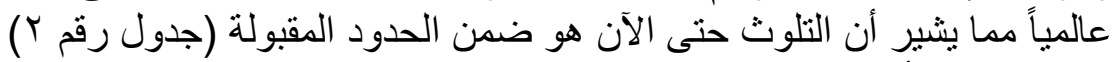

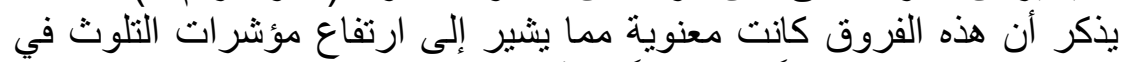

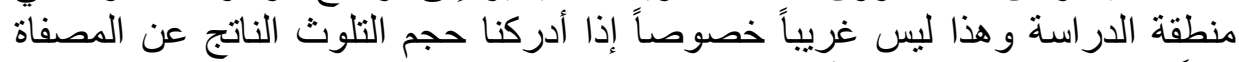
مثلاً و المنشآت الصناعية الأخرى و التي تبث سمومها في الجو ثم ينتقل بدوره للبيئة البهاء المحيطة كالتربة و الماء الماء.

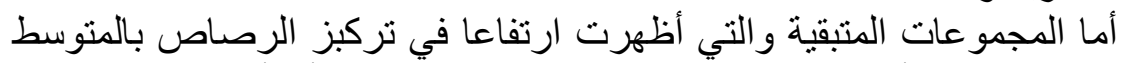

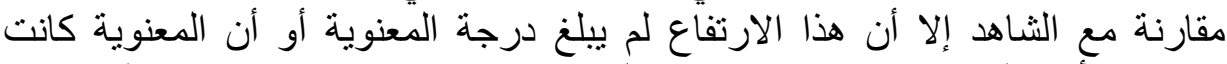

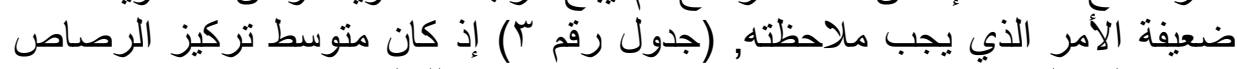

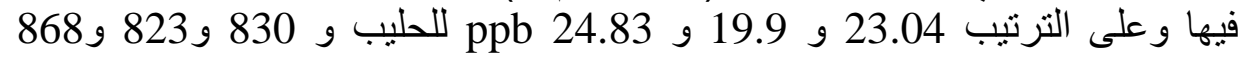
للام الأولى والثانيـة والرابعـة في حين كان في الثاهد 17.4 ppb 173

جدول رقمץ يتضمن نتائج تحليل الرصاص مقدرة بـ ppb

\begin{tabular}{|c|c|c|c|c|}
\hline الماء (ppb) التركيز في & المتوسط للام & للحليب (ppb) & العدد & المجمو عة \\
\hline$r, r$ & $V \wedge \pm V r V$ & $r, r \pm 1 \vee, \Sigma$ & $r$. & الثـاهد \\
\hline$r, r$ & $77 \pm V r$. & $r, \wedge \pm Y r$ & $r$. & المجمو عة (1) \\
\hline 1,7 & $\Lambda q \pm \wedge r r$ & $r, \tau \pm r$. & r) & المجمو عة(r) \\
\hline$v, l$ & $1 \cdots \pm q \vee r$ & $\wedge, \wedge \pm r q, \wedge$ & 19 & المجمو عة(r) \\
\hline$r, T$ & $\wedge ! \pm \wedge \uparrow \Lambda$ & 0,\rceil \pm Y \leq, \Lambda$ & 19 & المجمو عة (ع) \\
\hline
\end{tabular}

تبين من خلال الدراسة الإحصائية لنتائج تحليل عنصر الرعاص الرعاص وجود

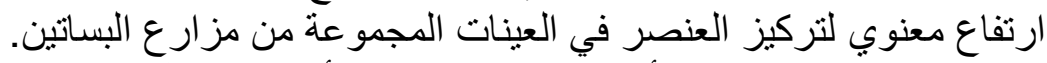

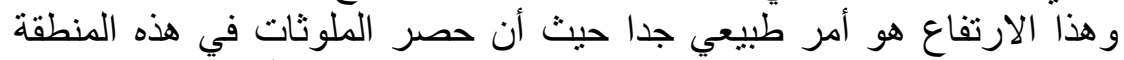

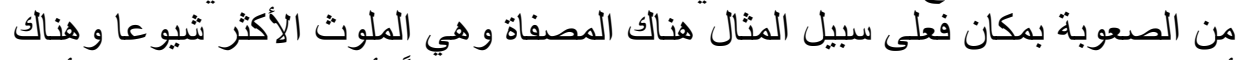

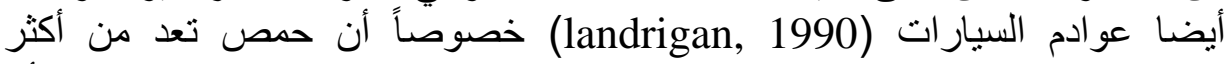

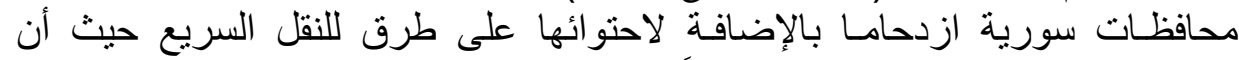

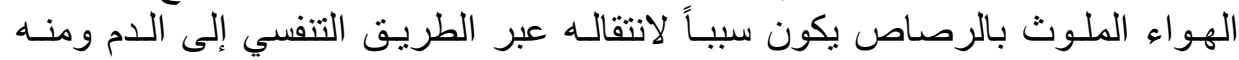


(Ludwig and Steigerwald, 1965; Chow and earl للحليب وكذلك بالنسبة للجلد (1970

مدكن أن يكون الهو اء الملوث سبباً لتلوث العلف الدريس و التبن و السيلاج والأعلاف المركزة

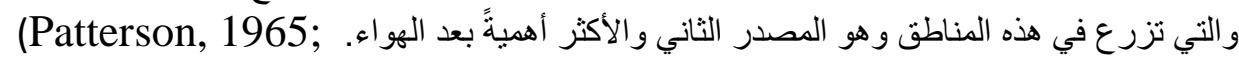
Delumyea and Kalivretenos, 1987; Nriagu and Pacyna, 1988;

Ducoffre et al., 1990)

بقي أن نذكر أن هنالك سبباً احتمالياً آخر يشارك في زيادة نركيز عنصر

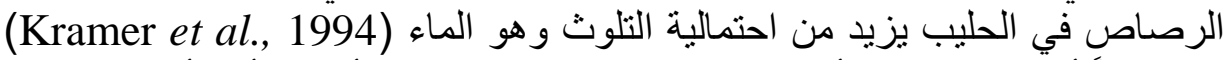

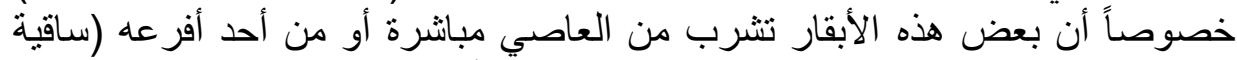

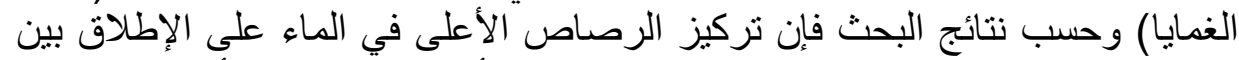

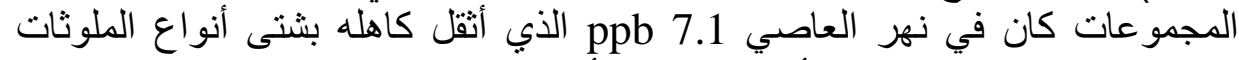

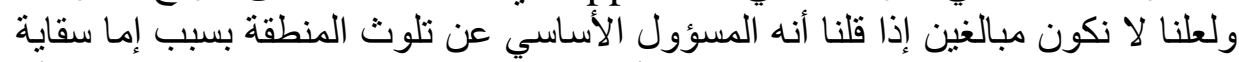

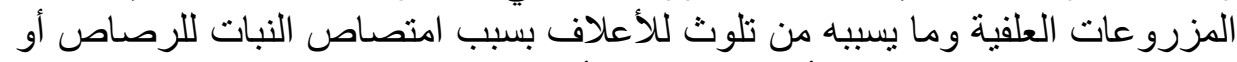

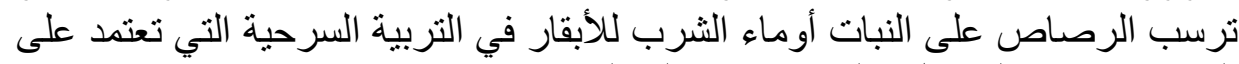

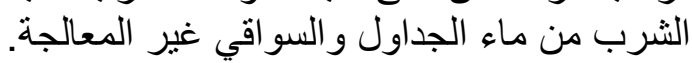

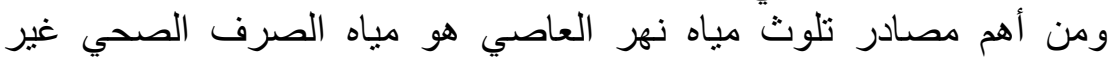

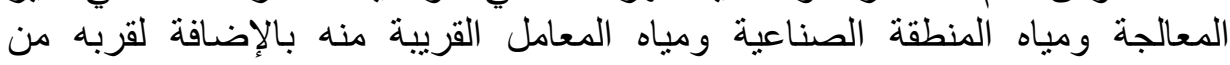
أما المنطقة التي تلي منطقة المجموعة الثالثة تلوثا فهي المجموعة الرابعة

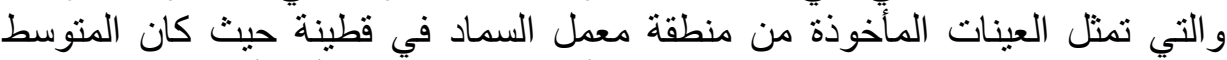

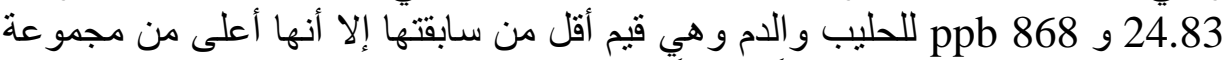

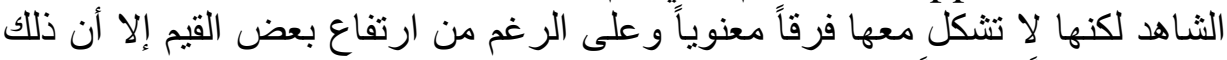

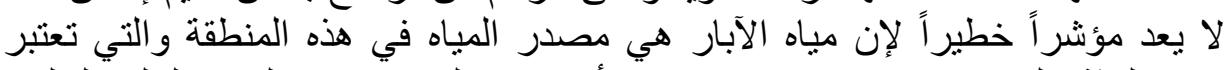

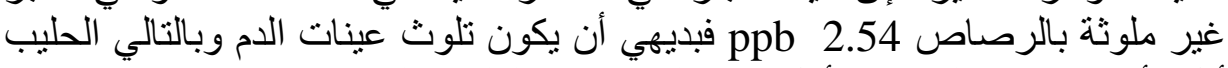

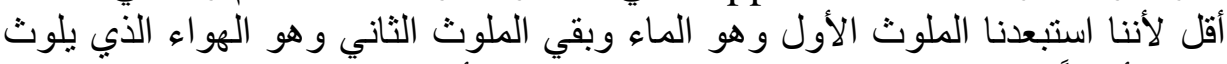

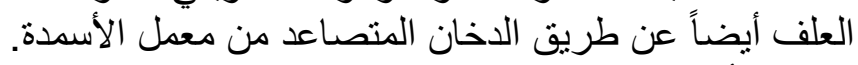

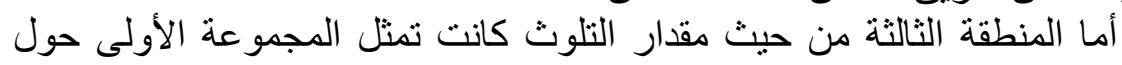

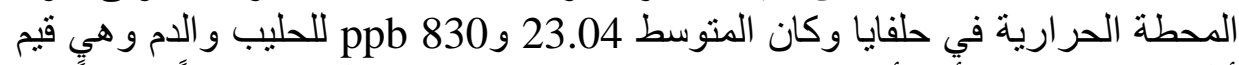

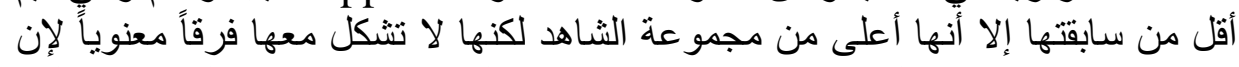

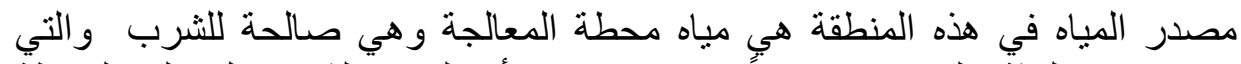

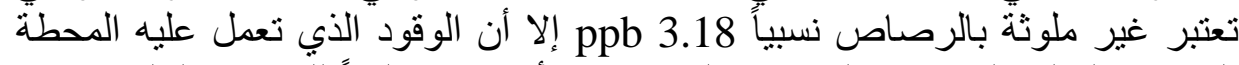

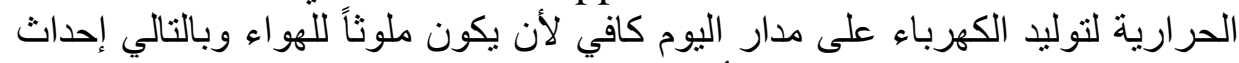
تلوث في البيئة كما سبق ونم شرحه أعلاه.

وأخيراً فإن المنطقة الرابعة من حيث مقدار التلوث كانت للمجموعة الثانية

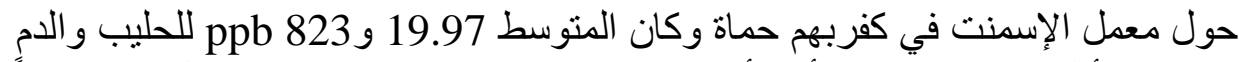

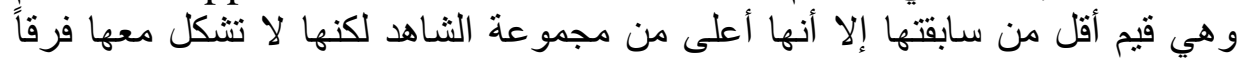




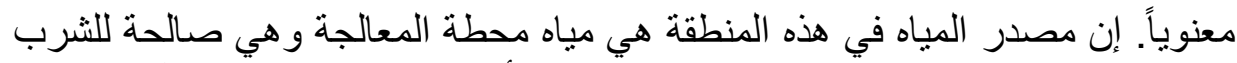
والتي تعتبر غير ملوثة بالرصاص فئ يعتبر ملوثاً للهو اء وبالتالي حدوث ملثئ تلوثا بالبيئة.

جدول رقم بّ: يوضح درجة المعنوية بين الثشاهد وبقية المجموعات

\begin{tabular}{|c|c|c|c|c|c|}
\hline المجموعة & المجمو عة" & المجمو عة ץ & المجمو عة| & الثـاهد & \\
\hline., $1 \leqslant \wedge$ & ${ }^{*}, \ldots$ & $\cdot, T \cdot V$ & . ror & - & الثاهد \\
\hline,,$\vee Y O$ & ${ }^{* *}, \ldots 7$ & ., OrV & - & , Y0T & المجمو عة| \\
\hline סזץ,. & ${ }^{* *},, \ldots 1$ & - & ., orv & $\cdot, T \cdot V$ & المجمو عة ب \\
\hline${ }^{* *} \cdot, ., 10$ & - & ${ }^{* *} \cdot, \ldots 1$ & ${ }^{* *} \cdot, \ldots 7$ & ${ }^{*}, \ldots$ & المجمو عةّ \\
\hline- & ${ }^{* *}, ., 10$ & סחז,. &., VYO &., $1 \leq 1$ & المجمو عة؛ \\
\hline
\end{tabular}

* فروق عالية المعنوية بين الثاهد و المجموعة الثالثة

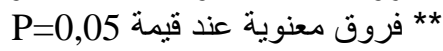

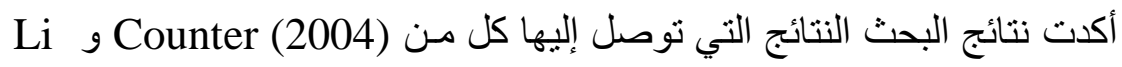

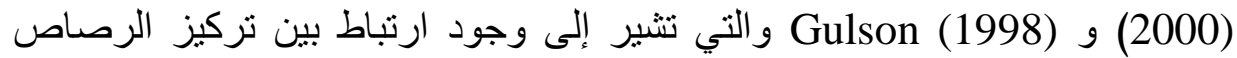

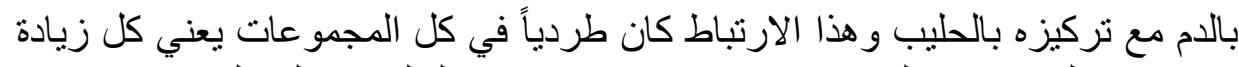

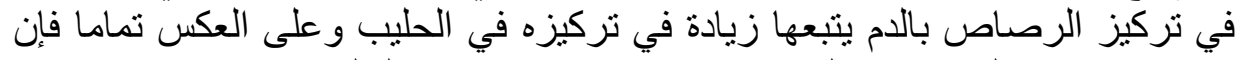

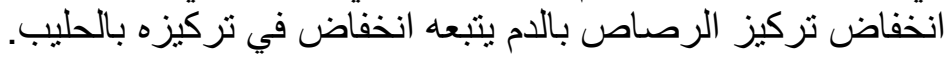

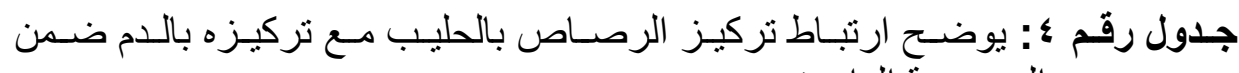

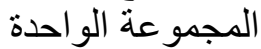

\begin{tabular}{|c|c|}
\hline درجة الآرتباط* & رقم المجمو عة \\
\hline$+\cdot, \vee V Y$ & الشـاهد \\
\hline$+\cdot, \wedge \Gamma \wedge$ & الأولى \\
\hline$+\cdot, q \cdot v$ & الثنانية \\
\hline
\end{tabular}


Assiut Vet. Med. J. Vol. 58 No. 132 January 2012

\begin{tabular}{|c|c|}
\hline$+\cdot$, · \ & الثالثة \\
\hline$+\cdot, 74 \Lambda$ & الر ابعة \\
\hline
\end{tabular}

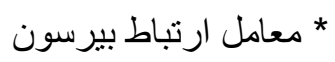

فقد كانت قيمة العلاقة بين تركيز الرصاص في الدم إلى تركيز الرصاص في

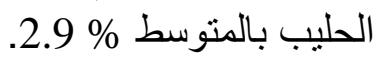

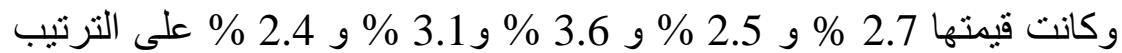

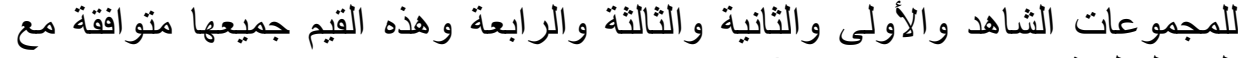
القيم العالمية (Counter, 2004)

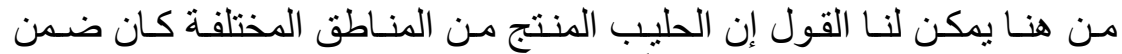

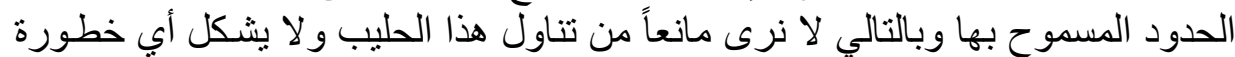

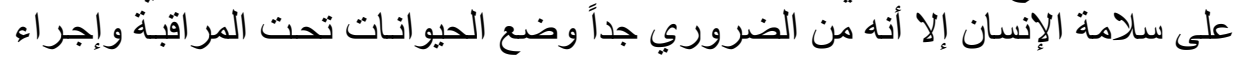
فحوصات دورية لمنتجات هذه الانه هن الحيو انات.

\section{المقترحات والتوصيات:}

- يمكن اعتماد قياس تركيز الرصاص في الحليب واعتباره كمؤشر لتركيز الرصاص - ضي ضرورة الفحص الدوري للاعصداص في حليب أو دم الأبقار خصوصا المرباة حول

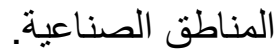
- ضرورة تحليل عينات من العلف والماء واءعداء وتحديد الحدود الدنيا المسموح بها واتخاذ

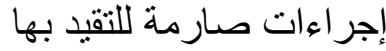
ـ التأكيد على وجود محطات معالجة مياه الصرف الصناء الصحي و المعامل قبل أن تصب في

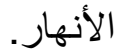
- التأكيد على وجود محطات تنقية للمياه قبل تقديمها للحيو ان(مياه صالحة للشرب).

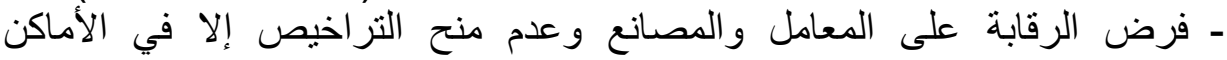
الصناعية. - منع الباعة الجو الين من بيع منتجات الحليب المكثوفة خصوصا للأطفال في المناطق

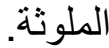

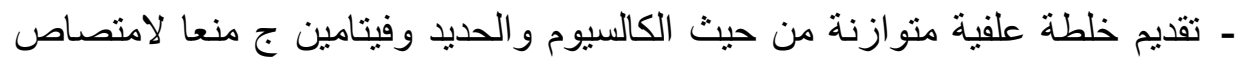

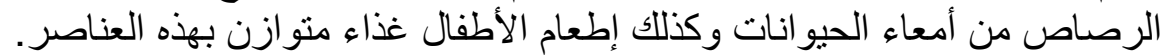

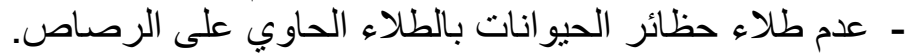

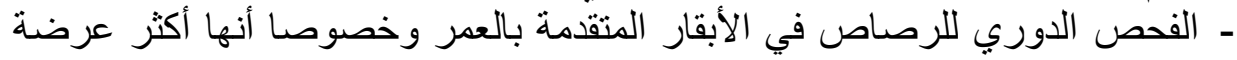
لسحب الرصاص من العظام بسبب ارتفع احتمالية الإصابة بمرض الإر حمى النفاس. 


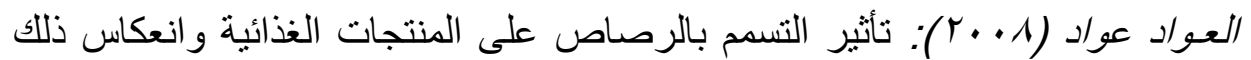

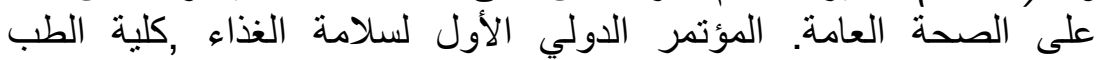
البيطري, جامعة البعث. (البه.

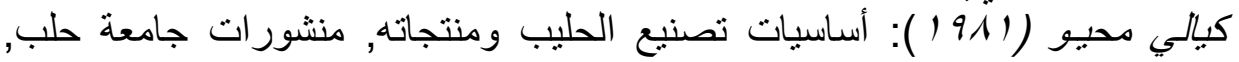
حلب, ص : :

\section{REFERENCE}

Barton, J.C.; Conrad, M.E.; Nuby, S. and Harisson, L. (1978): Effects of iron on the absorption and retention of lead. J. Lab. Clin. Med. 92: 536-547.

Centres For Disease Control, (1991): Preventing Lead Poisoning in Young Children. U.S Dept of Health and Human Services (Report), Atlanta, GA.

Chow, T.J. and Earl, J.L. (1970): Lead aerosols in the atmosphere: increasing concentrations. Science. Aug 7;169 (945): 577-580.

Counter, SA.; Buchanan, LH. And Ortega, F.J. (2004): Current pediatric and maternal lead levels in blood and breast milk in Andean inhabitants of a lead-glazing enclave. Occup Environ Med. Sep; 46(9): 967-73.

Delumyea, $R$. and Kalivretenos, A. (1987): Elemental carbon and lead content of fine particles from American and French cities of comparable size and industry Atmospheric environment, 21: 1643-1647.

Ducoffre, G.; Claeys, F. and Bruaux, P. (1990): Lowering time trend of blood lead levels in Belgium since. Environmental Research, 51: 25-34.

Farid, S.M.; Enani, M.A. and Wajid, S.A. (2004): Determination of Trace Elements in Cow's Milk in Saudi Arabia. Eng. Sci., 15, 2: 131-140.

Gulson, B.L.; Jameson, C.W.; Mahaffey, K.R; Mizon, K.J.N.; Patison, A.J.; Lwa, M.J.; Korsch and Salter, M.A. (1998): Relationships of Lead in Breast Milk to Lead in Blood, Urine, and Diet of the Infant and Mother. Environmental Health Perspectives. Volume 106, Number 10, October.

Jeng, S.L.; Lee, S.J. and Lin, S.Y. (1994): Determination of Cadmium and Lead in Raw Milk by Graphite Furnace Atomic Absorption Spectrophotometer. Journal of Dairy Science Vol. 77, No. 4. 
Hallen, I.P. and Oskarsson, A. (1995): Bioavailability of lead from various milk diets studied in a suckling rat model. BioMcmLv 8: 231-236.

Landrigan, PJ.; Silbergeld, EK.; Froines, JR. and Pfeffer, RM. (1990): Lead in the modern workplace. Am. J. Public Health. Aug., 80 (8): 907-908.

Li, PJ.; Sheng, YZ.; Wang, QY.; GU, LY. and Wang, YL. (2000): Transfer of lead via placenta and breast milk in human. Biomed Environ Sci. Jun; 13(2): 85-9.

Kramer, U. (1994): Die Bleibelastung Von Kindern Aus Ost- und Westdeutschland Expositionsquellen und Auswirkungen auf das Zentralnervensystem. Informatik, Biometrie und Epidemiologie, 25: 58-73

Levy, Y.; Zeharoa, A.; Grunebaum, M.; Nitzan, M. and Steinherz, R. (1985): Copper deficiency in infants fed cow milk. J. Pediatr., 106: 786-788.

Ludwig, J.H. and Steigerwald, BJ. (1965): Research In Air Pollution: Current Trends. Am. J. Public Health Nations Health. Jul., 55: 1082-92.

Nriagu, J.O. (1978): The Biogeochemistry of Lead in the Environment by Jerome O Biomedical Press, Amsterdam, New york, Oxford.

Nriagu, J.O. and Pacyna, J.M. (1988): Quantitative assessment of worldwide contamination ofair, water and soils by trace metals. Nature, 333: 134-139.

Oskarsson, A.; Jorhem, L.; Sundberq, J.; Nilsson, NG. and Albanus, L. (1992): Lead poisoning in cattle-transfer of lead to milk. Sci. Total Environ, 111: 83-94.

Patterson, C. (1965): Contaminated and natural lead environments of man. Archives of Environmental Health, 11: 344-360.

Pennigton, J.A.T.; Schoen, S.A.; Salmon, G.D.; Young, B.; Johnson, R.D. and Marts, R.W.J.E. (1995): Composition of core foods of the U.S. Food Supply, 1982-1991. III. Copper, manganese, selenium and iodine. J. Food Comp. Analysis, 8: 171-217.

Qiu, CAI.; Mei-Li Long; Ming, Zhu; Qing-Zhen Zhou; Ling Zhang and Jie Liu, (2009): Food chain transfer of cadmium and lead to cattle in a lead-zinc smelter in Guizhou, China. Environmental Pollution 157: 3078-3082.

Sharma, R.P.; Street, J.C.; Shupe, J.L. and Bourcier, D.R. (1982): Accumulation and depletion of cadmium and lead in tissues 
and milk of lactating cows fed small amounts of these metals. J. Dairy Sci. 65(6): 972-9.

Steijns, J.M. (2001): Milk ingredients as nutraceuticals. Int. J. Dairy Technol., 54, 81.

Walker, A. (1987): Trace Element Analysis, SAS, 10

WHO (2001): Regional Office for Europe, Copenhagen, Denmark, Chapter 6.7 Lead.

Wilhelm, M.; Lombeck, I.; Kouros, B.; Wuthe, J. and Ohnesorge, FK. (1995): Duplicate study on the dietary intake of some metals/metalloids by children in Germany. part II. Aluminum, cadmium and lead. Zentralblatt für Hygiene, 197: 357-369.

World Health organization, Geneva, (2000): IPCS-International Programme on Chemical Safety Contaminants.

Ziegler, EE.; Edwards, BB.; Jense, RL.; Mahaffet, KR. and Foman, SJ. (1978): Absorption and retention of lead by infants. Pediatr Res., 12: 29-34. 